\title{
CD4 Levels and Viral Load during Ulcerative Colitis Flares in Patients with Human Immunodeficiency Virus: A Case Series
}

\author{
Arroyo-Mercado Fray ${ }^{1}$, Likhtshteyn Michelle ${ }^{1}$, Huynh Chi Doan ${ }^{1}$, Chokshi Tanuj ${ }^{1}$, Chawla Gurasees S. ${ }^{1}$, \\ Grossman Evan $^{1,2}$, Ramirez Miguel ${ }^{1}$, Ojeda-Martinez Hector ${ }^{1,3}$, McFarlane Samy $\mathbf{I}^{1, *}$ \\ ${ }^{1}$ Department of Medicine, SUNY Downstate Health Sciences University, Brooklyn, New York \\ ${ }^{2}$ Division of Gastroenterology and Hepatology, SUNY Downstate Health Sciences University, Brooklyn, New York \\ ${ }^{3}$ Division of Infectious Diseases, SUNY Downstate Health Sciences University, Brooklyn, New York \\ *Corresponding author: Samy.mcfarlane@downstate.edu
}

Received May 10, 2019; Revised June 24, 2019; Accepted July 10, 2019

\begin{abstract}
Background: The "Remission Hypothesis" suggests that in patients with both HIV and UC an immunosuppressed state allows for the remission of UC and decreased number of flares. While the exact mechanism is unknown, this theory considers the relationship between CD4 count and flare progression. However, currently literature does not take into account the role that viral load might play in modulating flare activity. Methods: This is a case series including three patients with concurrent HIV and IBD at two large urban academic centers. A retrospective chart review was done and clinical information such as CD4+ count, viral load and flare symptoms were collected for each patient. Results: Three patients with a total of eleven UC flares were evaluated between the years of 2007 and 2018. Of the eleven flares, nine flares occurred while the viral load was undetectable, one flare occurred while the viral load was unknown, and one flare while the viral load was detectable. Conclusions: Nine out of eleven UC flares occurred while the patients' viral loads were undetectable, which can support a "Remission Hypothesis" which is inclusive of viral load instead of CD4+ count. However, it is important to note that the disease progression of Patient 3 does not completely support this version of the hypothesis. While we cannot comment on whether the "Remission Hypothesis" is true or not, we do believe a more inclusive theory including viral load should be considered.
\end{abstract}

Keywords: format, microsoft word template, style, insert, template

Cite This Article: Arroyo-Mercado Fray, Likhtshteyn Michelle, Huynh Chi Doan, Chokshi Tanuj, Chawla Gurasees S, Grossman Evan, Ramirez Miguel, Ojeda-Martinez Hector, and McFarlane Samy I, "CD4 Levels and Viral Load during Ulcerative Colitis Flares in Patients with Human Immunodeficiency Virus: A Case Series.” American Journal of Medical Case Reports, vol. 7, no. 8 (2019): 162-166. doi: 10.12691/ajmcr-7-8-3.

\section{Introduction}

Accumulating evidence indicates an association between CD4 count and Ulcerative colitis (UC) flares in patients with HIV $[1,2,3,4]$, whereby patients who have low CD4 counts are less likely to experience UC flares compared to those who have normal or elevated CD4 counts. While the exact mechanism is unknown, it is hypothesized that an immunosuppressed state allows for the remission of UC; this theory has been deemed the "Remission Hypothesis" [1]. It considers the relationship between CD4 count and flare progression; it does not, however, take into account the role that viral load might play in modulating flare activity.

Inflammatory Bowel Disease (IBD) is thought to be propagated by an exaggerated cell-mediated immune response to an antigen in a susceptible host [1]. IBD is characterized by increased activity of the gastrointestinal mucosal immune system [2]. This immune response is chiefly produced by T-Cells and B cells which recruit macrophages and neutrophils in order to further modulate a cytokine-mediated inflammatory process. CD4 T- Cells are the key cells which are being targeted in patients with Human Immunodeficiency Virus (HIV). Due to a comparable cell-mediated processes between HIV and IBD, it has been postulated that a concurrent HIV infection might have an impact on the immunopathology and prognosis of IBD [2]. The primary interest of our study is to evaluate whether CD4 count and viral load influence IBD activity.

\section{Materials and Methods}

This is a case series designed to evaluate patients with concurrent HIV and IBD at two large urban academic centers. We included all patients who were eighteen years of age or older that were seen at Downstate Medical 
Center (DMC) or Kings County Hospital (KCH). Patients to be included in this study were gathered using the International Classification of Diseases (ICD) 9 and 10. Data collection variables included demographics, laboratory and clinical data as well as procedure details, diagnoses, and complications. Our subjects were members of ethnic/racial minority groups and economically or educationally disadvantaged. Undetectable viral load was defined as the lower limit of detection of the assay use, viral load was measured by PCR. This was a case series with no investigator-participant interaction. Our institution does not require institutional board review for case series; however an IRB waiver was obtained.

\section{Case Series}

\subsection{Patient 1}

A 29-year-old African American male with a known diagnosis of both UC and HIV, compliant with antiretroviral therapy (ART), presented to the hospital with complaints of diffuse abdominal pain and bright red bloody diarrhea. His diarrhea had been occurring up to four times daily and had been ongoing intermittently for one month. He was diagnosed with UC at the age of 19, and his last exacerbation had been many years ago (exact time unknown). Examination findings upon presentation included abdominal tenderness to deep palpation in the upper left quadrant and pelvic region. Rectal exam revealed no ulcers or lesion and digital rectal exam was unremarkable, with no melena or bright red blood visualized. He was found to have a CD4 count of 420 cells/microliter and an HIV RNA viral load $<50$ RNA copies/milliliter. A colonoscopy during his admission revealed moderate pancolitis; lymphogranuloma venereum (LGV) and gonorrhea were ruled out. He was started on mesalamine while inpatient with observed clinical improvement; and was subsequently discharged home with initiation of mesalamine enemas along with continuous monitoring and outpatient follow up. Since his initial admission, the patient had three more UC flares over the next eight years, with CD4 count of 705 cells/microliters, 575 cells/microliter, and 320 cells/microliter on each respective flare, along with complete viral suppression (HIV viral load < 50 RNA copies/milliliter). This patient's HIV was well controlled at the time of all three episodes (Table 1 and Table 2).

During a three-year period between flares, the patient encountered multiple psychosocial barriers (undomiciled status, methamphetamine abuse, incarceration) and endorsed to his clinic providers that he stopped taking his ART. His CD4 count nadir during this time period was 155 cells/microliters, with viral load increased to 120,296 RNA copies/milliliters, placing him in the category of acquired immunodeficiency syndrome (AIDS). (Table 1) While he remained immunosuppressed for these three consecutive years, he experienced stable remission of UC symptoms. However, when patient was restarted on ART with appropriate immune response (CD4 > 200 cells/mL) and virologic suppression, he presented back to the hospital with relapse of UC, thereby suggesting a correlation between immune status, more specifically CD4 T cells count, and development of inflammation leading to IBD flares. Patient 1's clinical course supports the "Remission Hypothesis” with both CD4 Counts and viral load levels.

Table 1. Summary of Patients' CD4 Counts, Viral Loads, and Flares

\begin{tabular}{|c|c|c|c|c|c|c|c|c|c|}
\hline & Patient 1 & & & Patient 2 & & & Patient 3 & & \\
\hline Year & CD4 Count & Viral Load & Flare? & CD4 Count & Viral Load & Flare? & CD4 Count & Viral Load & Flare? \\
\hline 2007 & N/A & N/A & N/A & 372 & undetectable & Yes & unknown & unknown & Yes \\
\hline 2008 & N/A & N/A & N/A & 491 & undetectable & Yes & 577 & undetectable & Yes \\
\hline 2009 & 420 & undetectable & Yes & N/A & N/A & N/A & 577 & N/A & N/A \\
\hline 2010 & 436 & undetectable & No & 588 & undetectable & Yes & N/A & N/A & N/A \\
\hline 2011 & 501 & undetectable & No & N/A & N/A & N/A & 727 & undetectable & N/A \\
\hline 2012 & 705 & undetectable & Yes & N/A & N/A & N/A & 651 & undetectable & N/A \\
\hline 2013 & 757 & undetectable & Yes & 525 & detectable & Yes & N/A & N/A & N/A \\
\hline 2014 & 272 & detectable & No & 158 & undetectable & Yes & 645 & undetectable & N/A \\
\hline 2015 & 370 & detectable & No & 126 & detectable & No & 587 & undetectable & N/A \\
\hline 2016 & 155 & detectable & No & 527 & detectable & No & N/A & N/A & N/A \\
\hline 2017 & 200 & undetectable & No & N/A & N/A & N/A & 623 & undetectable & No \\
\hline 2018 & 320 & undetectable & Yes & 438 & detectable & No & 640 & undetectable & No \\
\hline
\end{tabular}

Table 2. Patient 1 Association between CD4 Count, Viral Load and Flares

\begin{tabular}{|l|l|l|}
\hline Patient 1 Flares & & \\
\hline Year & CD4 Count & Viral Load \\
\hline 2009 & 420 & undetectable \\
\hline 2012 & 705 & undetectable \\
\hline 2013 & 757 & undetectable \\
\hline 2018 & 320 & undetectable \\
\hline
\end{tabular}


Table 3. Patient 2 Association between CD4 Count, Viral Load and Flares

\begin{tabular}{|l|l|l|}
\hline Patient 2 Flares & & \\
\hline Year & CD4 Count & Viral Load \\
\hline 2007 & 372 & undetectable \\
\hline 2008 & 491 & undetectable \\
\hline 2010 & 588 & undetectable \\
\hline 2013 & 525 & 152,000 copies/microliters \\
\hline 2014 & 158 & undetectable \\
\hline
\end{tabular}

\subsection{Patient 2}

A 27-year-old African American female with a past medical history of sickle cell trait, iron deficiency anemia, and HIV, presented to clinic with a 3 week history of bloody diarrhea ( $>10$ bowel movements/day) following a one week course of clindamycin for a sinus infection. She was diagnosed with HIV in 2002 and endorsed compliance with her ART. Key examination findings upon initial presentation included hyperactive bowel sounds and mild tenderness in the left lower abdominal quadrant. Rectal exam showed small hemorrhoids with small amount of blood. On her admission, she was found to have CD4 count of 456 cells/microliter and HIV RNA viral load of $<400$ copies/microliters. Blood work showed elevated ESR and CRP while stool studies were negative for $C$. difficile toxin but significant for leukocytosis. A subsequent colonoscopy showed friable mucosa, diffuse erythema, and multiple ulcers suggestive of moderate UC. The patient was started on mesalamine and rowasa enema while inpatient with noted clinical improvement. She was then discharged on the same medication regimen with close follow up in the outpatient clinic. Three months after her initial diagnosis of UC, she became pregnant, stopped taking mesalamine and experienced several intermittent UC flares; CD4 nadir during this period was 491. She presented again three years later with repeated episodes of bloody diarrhea and underwent a repeat colonoscopy. Although the procedure was aborted midway due to severe inflammation, it showed an erythematous mucosa with loss of vascularity, numerous pseudopolyps in the left colon, and chronic inflammation. CD4 count during this flare was 158 cells/microliter and HIV RNA viral load was 20 copies/microliters. While, her CD4 count was suppressed during this admission, her viral load remained undetectable. Between 2007 and 2014, the patient had a total of five UC flares. Of these five flares, the patient's' viral load was undetectable on four occasions. Furthermore, her CD4 count remained above 250 cells/microliter during four of five flares. While the patient can be classified as having "controlled HIV" during her clinical course based on viral load, she did have a CD4 count nadir of 158 cells/microliter during one flare, as mentioned previously. The pattern of her CD4 count and viral load as they pertain to her IBD course are supportive of the "Remission Hypothesis".

\subsection{Patient 3}

A 62-year-old Hispanic male with known diagnosis of HIV, compliant with ART and virally suppressed, presented to the emergency department with complaints of abdominal pain and bright red blood per rectum. Patient was admitted to the hospital and underwent colonoscopy that showed severe inflammation from the splenic flexure to the rectum consistent with diagnosis of UC. He had a CD4 count of 577 cells/microliter and HIV RNA viral load <48 RNA copies/milliliter. Patient was initiated on infliximab and mesalamine treatment with significant clinical improvement. He was then discharged with appropriate outpatient follow up. One year later, he presented to clinic with complaints of 12 bloody and painful bowel movements per day for a duration of two weeks concerning for an UC flare. Colonoscopy showed pancolitis and pseudopolyps in the descending colon and rectum and biopsies were taken which ultimately revealed cytomegalovirus (CMV) colitis. CD4 count at the time was 495 cells/microliter. Although CMV gastrointestinal diseases are less common with CD4 count >50 cells/microliter, this patient's infection was likely from anti-TNF therapy secondary to his treatment of UC, which he had started one year prior. Patient was initiated on valganciclovir for CMV colitis, with clinical improvement; repeat colonoscopy showed moderate colitis, without evidence of active CMV infection. Throughout the next 10 years, this patient continued therapy with mesalamine, and experience no flares in his UC. CD4 nadir during these years was 587 cells/microliters and HIV viral load suppressed <20 copies/milliliters, thereby suggesting a negative association between immune status and development of UC flares (Table 1 and Table 4). While this patients disease progression does not support the "Remission Hypothesis", his initial presenting flare mentioned earlier does indeed support the hypothesis.

Table 4. Patient 3 Association between CD4 Count, Viral Load and Flares

\begin{tabular}{|l|c|c|}
\hline Patient 3 Flares & & \\
\hline Year & CD4 Count & Viral Load \\
\hline 2008 & 577 & undetectable \\
\hline
\end{tabular}

\section{Discussion}

The pathophysiology of HIV infection involves the virion attaching to and penetrating host $\mathrm{T}$ cells through CD4+ molecules and the chemokine receptor CCR5. Infected cells will then migrate to lymph nodes, where they can interact and potentially fuse with other CD4 cells, causing spread of HIV to deeper tissues. Gut associated lymphoid tissue (GALT) contains the largest reservoir of CD4 cells in the body, which also include a large pool of CCR5 expressing CD4 cells. As such, gut mucosa tissue represents a crucial target for acute HIV infection. Within 
3 weeks of acute HIV infection, more than half the CD4 T-cells in the lamina propria of the intestinal tract will have been destroyed. [4]

$\mathrm{T}$ cells play an important role in the inflammatory response in IBD, it has been postulated that the disease course can be altered by HIV infection. Yet, the management of patients with IBD and HIV remains a clinical challenge as the pathophysiology and clinical course of concomitant conditions remains unclear. The consensus on how to treat these patients is also uncertain. Hypothetically, low levels of CD4 cells due to an HIV infection could lead to remission of IBD, a phenomenon that is referred to as the "Remission Hypothesis," but the data supporting this theory has been inconsistent. Patients with CD4 counts above 200 cells/microliter are classified as immunocompetent which in theory makes them prone to develop more flares than patients with CD4 counts below 200 cells/microliter. [1]

The number of patients selected for this case series was limited due to the uncommon coexistence of these conditions. Patient 1 had multiple IBD flares while maintaining an undetectable viral load, and a CD4 count above 250 cells/microliter. (Table 2). He was in stable remission of IBD for 3 years during which time he was immunosuppressed with a CD4 nadir of 155 cells/microliter (Table 1); his clinical course favors the remission hypothesis. Similarly, Patient 2 had five flares between 2007 and 2014, and four of them are congruent with the "Remission Hypothesis"; with only one flare occurring while the patient had a detectable viral load, deeming the patient immunocompromised. Unlike Patients 1 and 2, Patient 3 had one flare between 2007 and 2018, during which his HIV was well controlled. However, he remained well controlled throughout his disease course until 2018 and had no further flares. Patient 3 does not completely support the "Remission Hypothesis", however as mentioned previously his UC was diagnosed while his HIV disease was well controlled.

A review of the literature indicates that there are very few studies on patients with IBD and HIV co-infection, with most of the patients being individual case reports. In a systematic review of literature, a total of 9 case reports in English, between 1984 and 2009 were found; 7 reports refuted the Remission Hypothesis, while 2 reports did not make any conclusions [1]. On the other hand, two case control studies and two case series published between 1996 and 2009 all supported the Remission Hypothesis. One case series described 4 cases of patients with CD who became infected with HIV and experienced a stable remission for the next $5,8,8$, and 8 years respectively [5] These different studies explored the relationship between IBD and CD4 count, however they do not mention or draw any conclusions regarding the role viral load plays in IBD modulation. Whereas, in our patient analysis, we considered the significance of viral load during IBD flares.

It's important to consider viral load because is the standard used to determine HIV disease activity; and is the first to respond to effective therapy with an immediate decline, compared to CD4 count that shows a slower trend towards normalization [6]. It is worth mentioning that since acute HIV infections can often present with diarrhea, it makes the diagnosis of IBD or IBD flare challenging. When faced with a patient with AIDS (CD4 count $<200$ cells/mL) and gastrointestinal symptoms, it is important to consider AIDS related pathology and opportunistic infections, in the differential diagnosis to IBD. This is in contrast to a more immunocompetent patient presenting with IBD symptoms, in which case the etiology is more likely to be from IBD than it is to be HIV related. Patient 3 from our case series was found to have CMV colitis, although his CD4 count at the time was 495 cells/mL. Although CMV gastrointestinal diseases are more commonly seen in AIDS patient with a CD4 count $<50$ cells/microliter, this patient's infection was attributed to the immunomodulation secondary to treatment of UC, which he had started one year prior. This infers that differentiating IBD flare from opportunistic infection can at times be difficult, and colonoscopy might be required in order to differentiate an IBD flare from an opportunistic infection.

Furthermore, perhaps CD4 cell counts are not the appropriate primary marker to use when trying to understand the relationship of HIV infection to the disease course of IBD. One study postulated that it was not the absolute number of CD4 cells that affected the relapse rate of Crohn's (CD) but rather the functional ability of the CD4 cells [7]. When comparing the number of CD4 cells in the lamina propria of patients with CD to normal control, both groups were found to have similar numbers. However, the CD4 cells in CD patients were shown to secrete more of the cytokine IL-2, which indicates $\mathrm{T}$ cell activation, thereby suggesting a higher functional ability of CD4 cells in CD when compared to healthy control [7].

While our case series has several limitations including a small sample size, no racial diversity, and reliance on documentation and coding, it is the first study that considers viral load in addition to CD4 count when considering IBD flares and disease progression. Our three patients had significant episodes of flare with relatively normal to high CD4 count and suppressed viral load, which raises the question as to whether IBD flares occurs as a consequence of CD4 count above 250 or high viral load. While the exact mechanism involved in flare of IBD in HIV populations remains unclear, large retrospective as well as prospective cohort studies are needed to elucidate such mechanisms and other risk factors involved in flare with the possibility of providing data that would help strategize regarding the optimal therapeutic approach for this vulnerable HIV populations with IBD flares.

\section{Summary}

What is already known about this subject:

- An association between CD4 count and UC flares in patients with HIV; patients who have low CD4 counts are less likely to experience UC flares compared to those who have normal or elevated CD4 counts.

- CD4 T- Cells are the key cells which are being targeted in patients with HIV. Due to a comparable cell-mediated processes between HIV and IBD, it 
has been postulated that a concurrent HIV infection might have an impact on the immunopathology and prognosis of IBD

- Some case series and case reports have shown conflicting evidence regarding support for the Remission Hypothesis

What are the new findings:

- While there may indeed be a relationship between CD4 count and UC flare progression, and ultimately remission, HIV viral load should be considered and further studied

- HIV viral load is a more accurate and more important marker of HIV disease progression and therefore a relationship between viral load and UC flare needs to be further studied

\section{Acknowledgements}

This work is supported, in part, by the efforts of Dr. Moro O. Salifu M.D., M.P.H., M.B.A., M.A.C.P., Professor and Chairman of Medicine through NIH Grant number S21MD012474.

\section{References}

[1] Skamnelos A, Tatsioni A, Katsanos KH, Tsianos V, Christodoulou D, Tsianos EV. CD4 count remission hypothesis in patients with inflammatory bowel disease and human immunodeficiency virus infection: a systematic review of the literature. Annals of Gastroenterology: Quarterly Publication of the Hellenic Society of Gastroenterology. 2015; 28(3): 337-346.

[2] Stephan R. Targan, Fergus Shanahan and Loren C. Karp (eds.) Inflammatory Bowel Disease: From Bench to Bedside, 2nd Edition, 863-873.

[3] Abramowitz, M., Ramachandran, R., Koczka, C., \& Lawlor, G. (2013). P-068 YI Human Immunodeficiency Virus: CD4 Count Does Not Impact Ulcerative Colitis Relapse Risk. Inflammatory Bowel Diseases, 19.

[4] Adiga A, Panikkath D, Nugent K (2016) A Review of Inflammatory Bowel Disease in Patients with Human Immunodeficiency Virus Infection. J AIDS Clin Res 7: 575.

[5] Pospai D, Rene' E, Fiasse R, et al. Crohn's disease stable remission after human immunodeficiency virus infection. Dig Dis Sci. 1998; 43: 412-419.

[6] Shoko C, Chikobvu D. A superiority of viral load over CD4 cell count when predicting mortality in HIV patients on therapy. BMC Infectious Diseases. 2019; 19(1).

[7] Lautenbach E, Lichtenstein GR. Human immunodeficiency virus infection and Crohn's disease: the role of the CD4 cell in inflammatory bowel disease. J Clin Gastroenterol. 1997; 25: 456-459.

(C) The Author(s) 2019. This article is an open access article distributed under the terms and conditions of the Creative Commons Attribution (CC BY) license (http://creativecommons.org/licenses/by/4.0/). 